\title{
AMTI FILTER DESIGN FOR RADAR WITH VARIABLE PULSE REPETITION PERIOD
}

\author{
Michal Řezníček — Pavel Bezoušek — Tomáš Zálabský *
}

\begin{abstract}
This paper presents a design of a Doppler AMTI filter, for a radar with a variable pulse repetition period (stagger). The filter can suppress ground and volume clutter echoes simultaneously. The maximum filter impulse response length is limited to 5 coefficients due to a limited radar system stability and a radar antenna movement.
\end{abstract}

K e y w or d s: filter, frequency response, AMTI, FIR, MTI, impulse response, clutter, Doppler processing

\section{INTRODUCTION}

This work deals with a simple ground and volume clutter cancelation in monostatic radar with a variable pulse repetition period (stagger). The impulse response length is limited to a maximum of 5 coefficients according to the client's requirements. This short filter is particularly suitable for semi-coherent (magnetron) radars with a low frequency stability, or where the antenna beam intercepts only a few impulse echoes. The processed data is in the form of a signal complex envelope. The data was provided by the company RETIA Inc.

The clutter consists of both the received echoes scattered from a static ground objects with zero Doppler frequency and the volume clutter of echoes scattered from moving meteo-objects with nonzero Doppler frequency (Fig. 1). To detect and track airplanes or helicopters both clutter types should be extracted from the received signal. In history this problem has been solved using analog filters with two stop bands. Nowadays thanks to a high processors computation performance available, it is possible to solve this problem using digital Doppler filters.

\section{CLUTTER PARAMETERS}

In Fig. 1 a transmitted pulse is scattered back by three typical objects: by a moving airplane, a moving meteoclutter and a static ground clutter. The frequencies of the echoes are shifted by Doppler frequencies $\omega_{d}[1,5,7]$, which in the discrete time domain correspond to $\Omega_{d}$ :

$$
\omega_{d}=-4 \pi \frac{v_{d}}{\lambda} ; \quad \Omega_{d}=T_{o p} \omega_{d}
$$

where is $v_{d}$ is the radial component of the scattering object velocity, $T_{o p}$ is a pulse repetition period and $\lambda$ is the transmitted signal wavelength.

The discrete time complex envelope spectrum of the echo from static and moving objects is shown in the Fig. 2.
It is evident that to suppress both clutter types we need two stopbands: one at the zero frequency and the other at the meteo-clutter Doppler frequency $\Omega_{d}$.

The power and the Doppler frequency of a real signal are shown in Figs. 3 and 4 where it is possible to note highly correlated echoes from a ground clutter and meteoclutter. The depicted signal was received during one antenna revolution. The presented data were provided by the company RETIA Inc.

\section{AMTI FILTERS STRUCTURES}

In the literature, two types of AMTI filter are described. The first structure is demonstrated in Fig. 5. This "real AMTI" filter suppresses the both clutter types in one step. The second type - the "cascaded AMTI" shown in Fig. 11 suppresses the ground clutter in the first step and the meteo-clutter in second step after the signal frequency shift by the meteo-clutter Doppler shift [1].

\subsection{Real AMTI filter}

The structure of the real AMTI filter is illustrated in e Fig. 5. At first the signal entering the filter is shifted by the half of the meteo-clutter Doppler frequency $\Omega_{d} / 2$ so that the ground and meteo-clutter spectra are symmetrically displaced around the zero frequency. Now it is possible to use a filter with symmetrical stopbands at $\pm \Omega_{d} / 2$ frequencies. Such a transfer characteristics can be realized using a real filter with a cosine transfer function approximation keeping a constant group delay. If the group delay depends on the signal (Doppler) frequency the target range evaluated from the signal time delay is determined with a lower accuracy [1].

For the power transfer function $H(\Omega)$ of the real AMTI filter it holds [2]

$$
|H(\Omega)|^{2}=\left[b_{2}+2 b_{1} \cos \Omega+2 b_{0} \cos (2 \Omega)\right]^{2}
$$

\footnotetext{
* Department of Electrical Engineering, University of Pardubice, Studentska 95, 53210 Pardubice 2, Czech Republic michal.reznicek@student.upce.cz, pavel.bezousek@upce.cz, tomas.zalabsky@student.upce.cz
} 


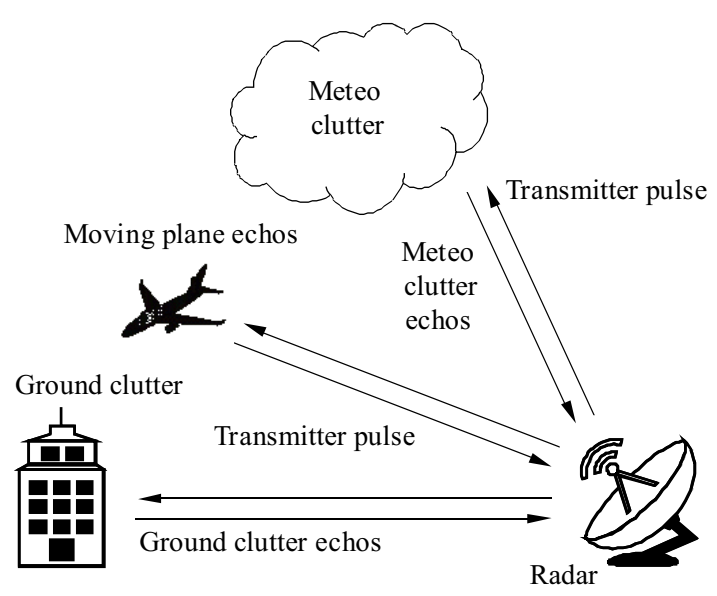

Fig. 1. Transmitted and received signal

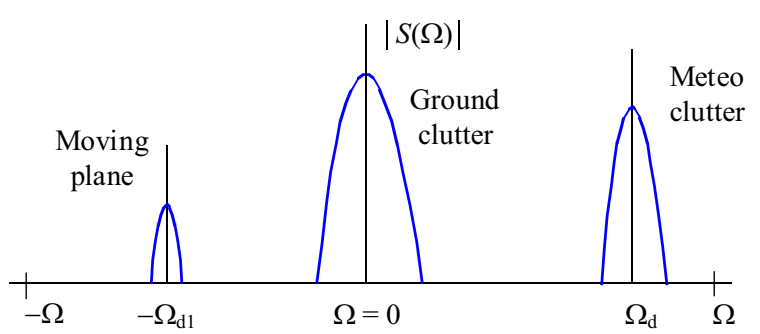

Fig. 2. The complex envelope spectrum of the received signal

where $\left\{b_{0}, b_{1}, b_{2}\right\}$ are the filter coefficients. Using the following equality [3]

$$
\cos (2 \Omega)=2 \cos ^{2}(\Omega)-1
$$

we get

$$
|H(\Omega)|^{2}=\left[b_{2}+2 b_{1} \cos \Omega+2 b_{0}\left(\cos ^{2}(\Omega)-1\right]^{2}\right.
$$

After substitution of

$$
\cos \Omega=x \ldots \text { if } \Omega \in(0, \pi\rangle \rightarrow x \in\langle-1,1\rangle
$$

into (4) and a necessary adjustment, it is possible to get

$$
|H(x)|^{2}=\left[\left(b_{2}-2 b_{0}\right)+2 b_{1} x+4 b_{0} x^{2}\right]^{2} .
$$

Zeros of $|H(x)|^{2}$ are the same as zeros of the second order polynomial

$$
H(x)=\left[\left(b_{2}-2 b_{0}\right)+2 b_{1} x+4 b_{0} x^{2}\right] .
$$

The next step is to express $H(x)$ as a product of root factors

$$
H(x)=\left(x-x_{1}\right)\left(x-x_{2}\right)=x_{1} x_{2}-\left(x_{1}+x_{2}\right) x+x^{2},
$$

where $x_{1}, x_{2}$ are the roots of $H(x)$.
Comparing coefficients at the corresponding powers of $x$ in (7) and (8) we get

$$
\begin{aligned}
& b_{0}=0.25, \\
& b_{1}=-0.5\left(x_{1}+x_{2}\right), \\
& b_{2}=x_{1} x_{2}+2 b_{0} .
\end{aligned}
$$

The polynomial $H(x)$ in (8) has always two roots, either real or complex conjugated. For the clutter suppression we need at least one real root (for example $x_{1}$ ) near to

$$
x_{1}=\cos \frac{\Omega_{d}}{2} .
$$

Then the second root $x_{2}$ should be also real. For finding of the second root, we can use one of the two following attitudes: As we need to create the notches only at Doppler frequencies of the ground clutter and the meteoclutter, then either $\left|x_{2}-x_{1}\right|=\Delta x \ll 1$ (close roots) or $\left|x_{2}\right|>1 \ldots$ then $H(\Omega)=0$ will have only one real solution in the interval $\Omega \in\langle 0,2 \pi\rangle$ (distant roots).

\subsubsection{Real AMTI filter with the "distant roots"}

In the case of "distant roots" we require: $\left|x_{2}\right|>1$. The calculation sequence should be as follows:

- Calculation of the discrete time Doppler angular frequency $\Omega_{d}=2 \pi F_{d}$ and the first root $x_{1}=\cos \left(\Omega_{d} / 2\right)$, where $F_{d}=\omega_{d} T_{o p}$

- Selection of an auxiliary variable $x_{3} \in\langle-1,1\rangle ; x_{3} \neq 0$.

- The second root calculation: $x_{2}=1 / x_{3}$; (then $\left.\left|x_{2}\right|>1\right)$.

The examples of the AMTI filter characteristics with distant roots with different position of the second root $x_{2}$ are shown in Fig. 6. It is obvious that an inappropriate choice of the second root position could create warped characteristics in the internal passband (ie in the band between the notches in Fig. 6). To overcome this problem additional optimization conditions should be used. The first one is not to allow the transfer function to drop in this band. The convenient indicator of this condition could be the first derivative sign. If this sign changes more than twice in the internal passband it is evident that the characteristics is bent. The second condition requires balanced parameters outside the stop band as described in equation

$$
|H(\Omega=0)|=|H(\Omega=\pi)| .
$$

The example of an optimized characteristics is shown in Fig. 7. Here we selected the best response without any warping and with a maximum balance outside the stop band. 


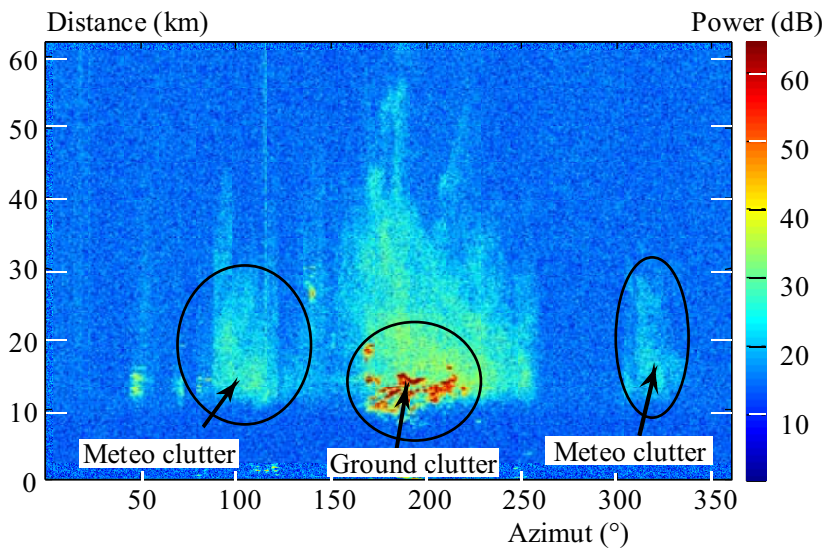

Fig. 3. Clutter power in the real received signal

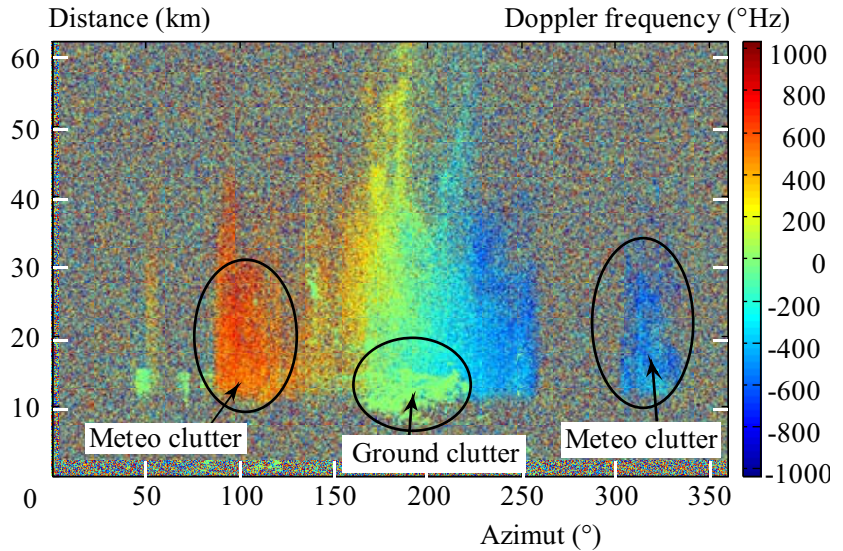

Fig. 4. Clutter Doppler frequency in the real received signal

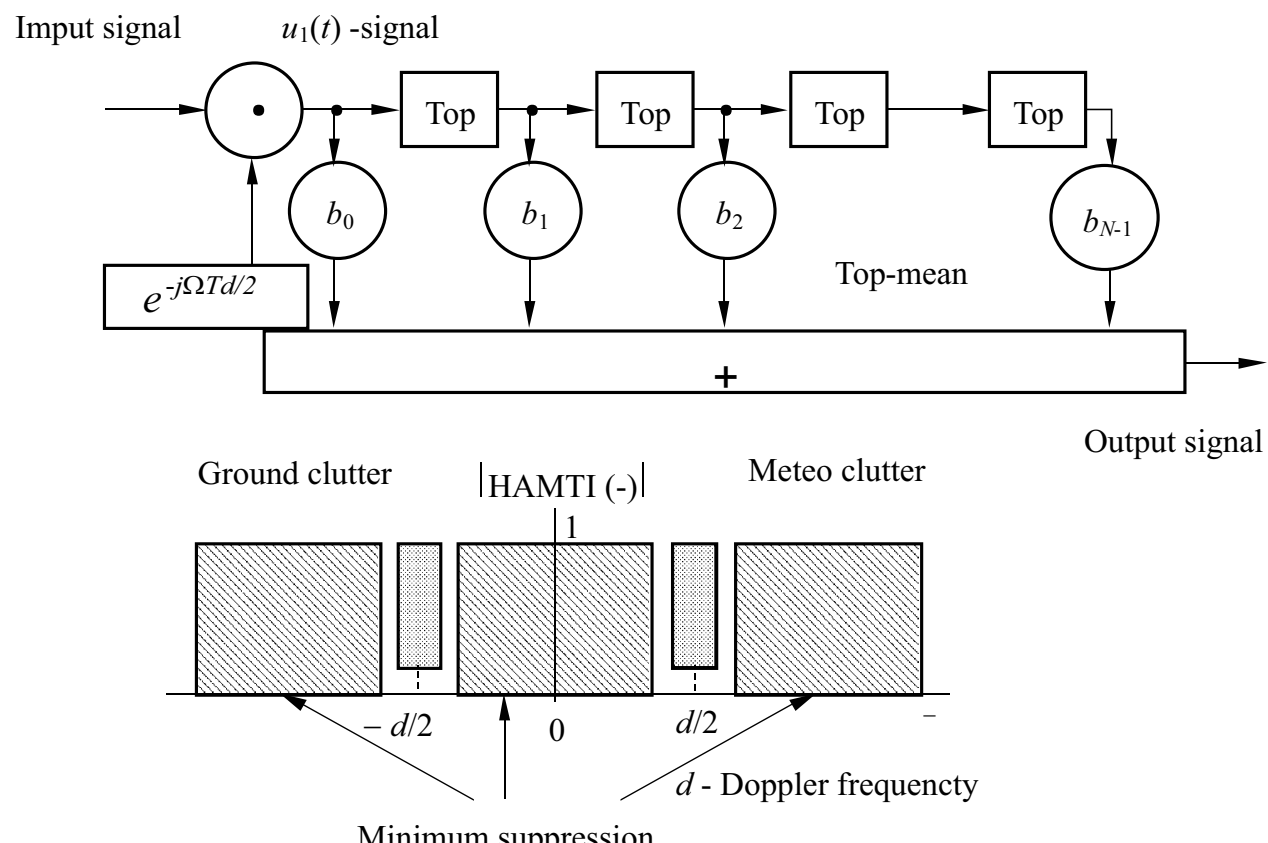

Minimum suppression

Fig. 5. Structure of the real AMTI filter

\subsubsection{Real AMTI filter with "close roots"}

In this case we are looking for two near solutions of the equation $H(\Omega)=0$ in the range of $\Omega \in\langle 0, \pi\rangle$. Then: $\left|x_{2}-x_{1}\right| \equiv \Delta x \ll 1$. The calculations sequence should be as follows:

- Calculation of the discrete time angular Doppler frequency $\Omega_{d}=2 \pi F_{d}$ and $x_{10}$, the mean value of the $x_{1}$ and $x_{2}: x_{10}=\cos \left(\Omega_{d} / 2\right)=0,5\left(x_{1}+x_{2}\right)$, where $F_{d}=\omega_{d} T_{o p}$.

- Selection of a positive value $\Delta x \ll 1$ (the roots $x_{1}$ and $x_{2}$ distance).

- Calculation of the roots: $x_{1}=x_{10}-\frac{\Delta x}{2}, x_{2}=x_{10}+\frac{\Delta x}{2}$.

The example of the power transfer function of a real AMTI filter with two close roots is displayed in Fig. 8. This filter advantage is in the possibility to adapt the stopband width to the clutter spectra requirements changing the roots distance.

3.1.3 Effect of the stagger on frequency response of a real AMTI filter

Now we will study the impact of a variable sample period on a depth of the filter suppression notches. It could be expected that the stopbands depths will be worse for a staggered signal then for a signal with a constant sample period.

To analyze the impact of the stagger on the filter transfer two matrices containing two models of complex envelopes of the input signals with constant amplitudes and variable Doppler frequencies were created. In the rows of each matrix signal samples of a constant Doppler frequency $\left(\Omega_{m} \in\left\langle 0 ; \Omega_{d} / 2\right\rangle\right)$ are successively ordered. In 


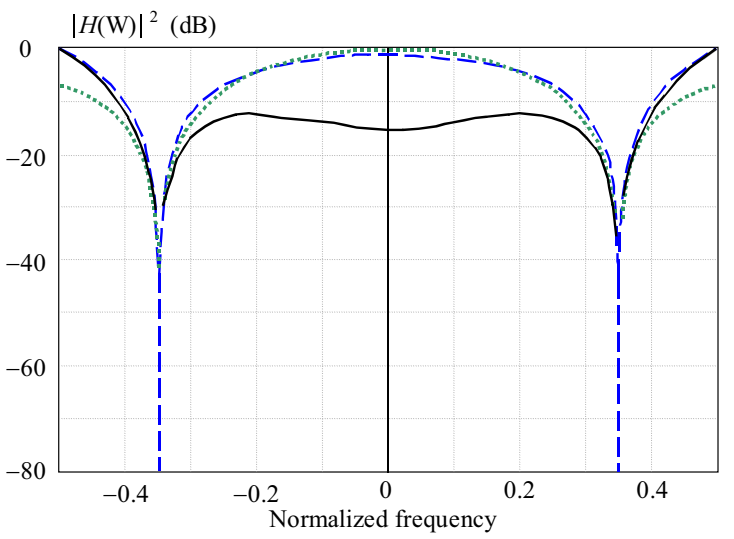

Fig. 6. frequency response for AMTI with "distance roots"

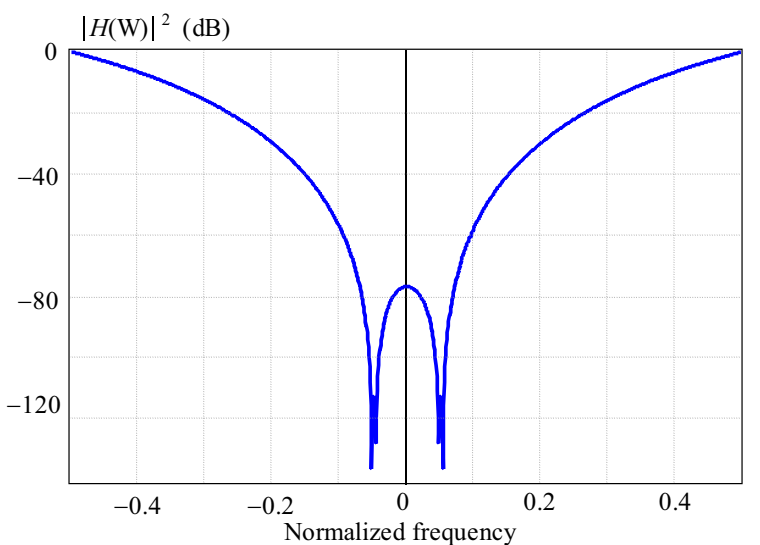

Fig. 8. Frequency response of the AMTI filter with "close roots"

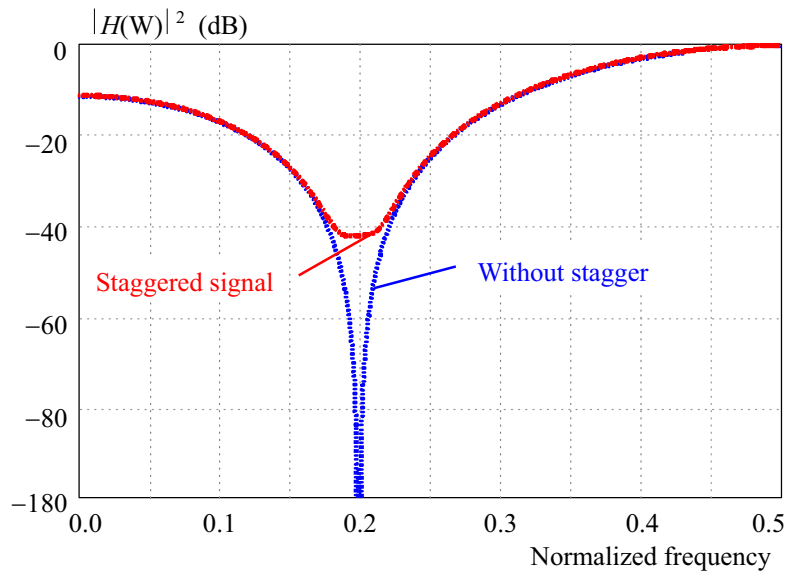

Fig. 10. Frequency response of an AMTI filter with "close roots" for a staggered and non-staggered period signal

the first matrix the time distances $T_{o p}$ between the adjacent samples are constant (the non-staggered signal). In the second matrix the sample distances are variable: $T_{\text {variable }}=T_{o p} \pm d T$ where $d T$ is a random variable in the range of $\left\langle-0,01 T_{o p} ; 0,01 T_{o p}\right\rangle$ with a uniform distribution (the staggered signal). The both signals undergo the AMTI filtration and filter transfers for a staggered and non-staggered signals are computed.

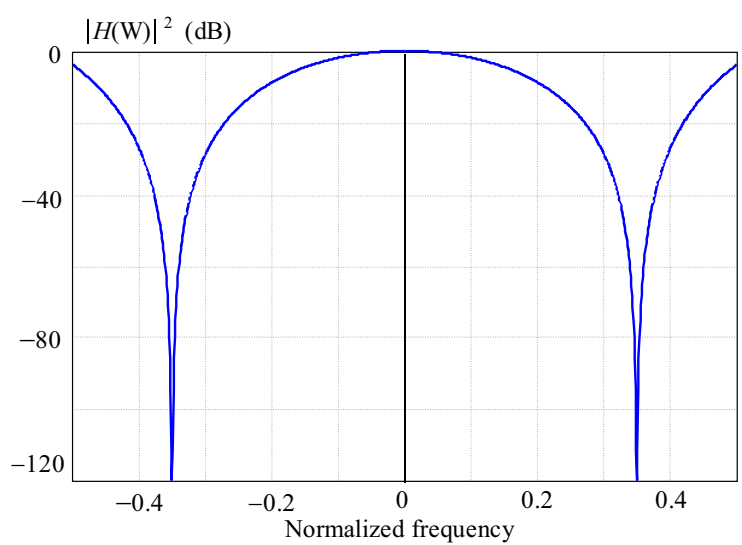

Fig. 7. frequency response for AMTI with "distance roots" after optimization

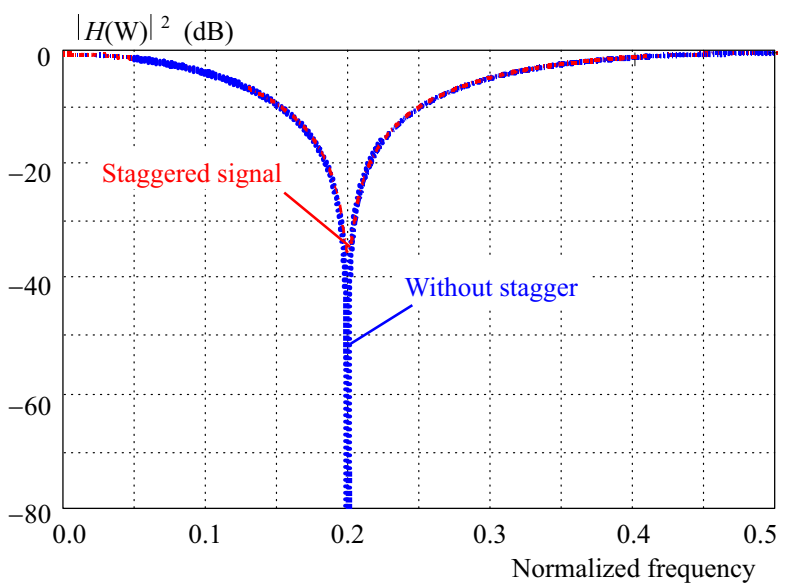

Fig. 9. Frequency response of an AMTI filter with "distant roots" for a staggered and non-staggered period signal

Figure 9 compares frequency responses of the AMTI filter with distant roots for the staggered and for the constant sample period signal. It is evident that in the stopband the staggered period signal characteristics is much worse than that of the constant period one. Figure 10 displays the same situation for an AMTI filter with "close roots". We can see, that in this case the suppression deterioration due to the stagger is less significant.

\section{CASCADED AMTI FILTER}

This structure consists of two cascaded MTI filters (Fig. 11). Each MTI filter has one stop band at zero Doppler frequency for clutter suppression. After ground clutter suppression in the first filter the signal frequency is shifted by the Doppler frequency $\Omega_{d}$ of the moving meteo-clutter so that its power spectrum will appear at zero frequency again. And then it is suppressed using the second MTI filter [1].

This MTI filter pulse response is described by the following equation $[4-6]$.

$$
H_{M T I}(t)=\delta(t)-2 \delta\left(t-T_{o p}\right)+\delta\left(t-2 T_{o p}\right),
$$

where $T_{o p}$ is the (constant) radar repetition period. 


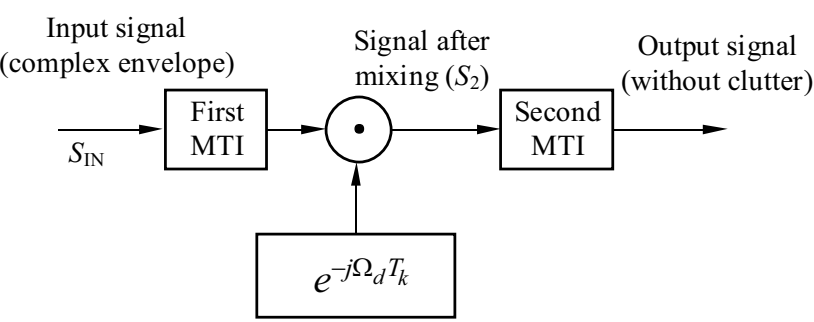

Fig. 11. Cascaded AMTI filter
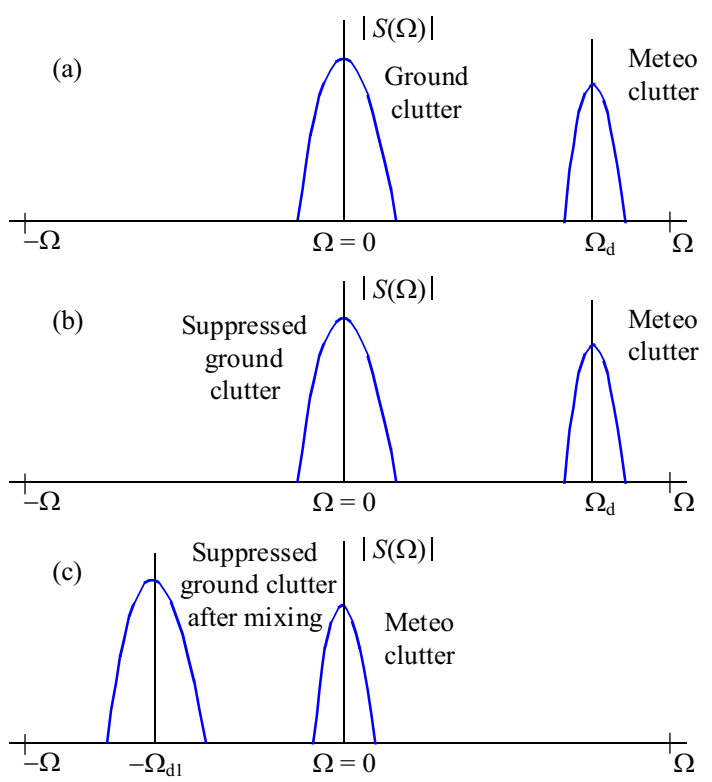

Fig. 12. spectrum complex envelope before and after frequency shift

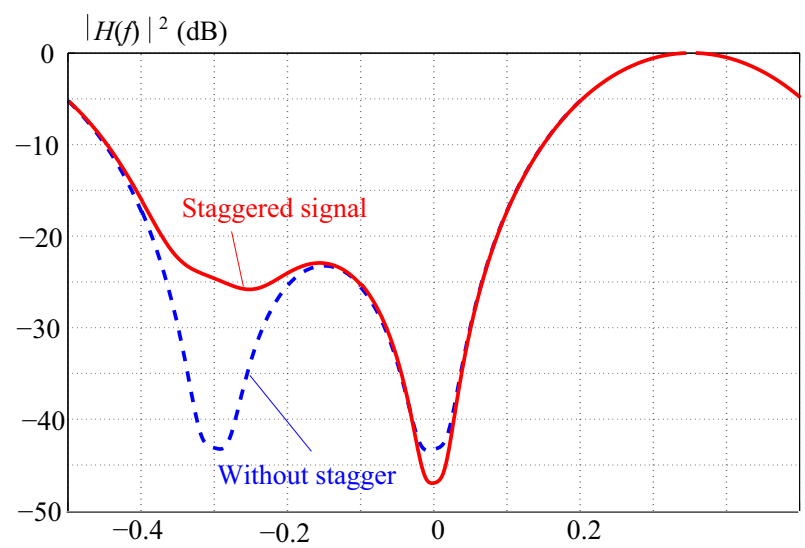

Fig. 13. spectrum complex envelope before and after frequency shift

Fig. 12a displays the spectrum of the input signal at the first MTI filter and in Fig. 12b the spectrum of $s_{2}(t)$ after frequency shifting is shown. The frequency shift is described using the following equation

$$
\left|S_{2}(\Omega)\right|=\left|S_{i n}\left(\Omega-\Omega_{d}\right)\right| \rightarrow S_{2}(t)=e^{-j \Omega_{d} t} s_{i n}(t),
$$

where $S_{\text {in }}$ is the spectrum of the signal complex envelope at the mixer input and $S_{2}$ is the spectrum at its output shifted by the Doppler frequency $\Omega_{d}$.
Cascaded AMTI filter characteristics are shown in Fig. 13. It is evident that the cascaded AMTI filter has deep and relatively wide stop bands. But in the case of a staggered period signal the suppression of the meteoclutter is very poor.

The anticipated reason of this effect could be a great uncertainty of time distances between signal $s_{2}$ samples after the first MTI filtration since every sample at the first MTI filter output is a combination of three input samples with various time separations.

\section{AMTI FILTER REAL DATA TESTING}

Figure 14 illustrates a power and a Doppler frequency of a real received signal during one radar antenna revolution at a particular distance before and after filtration by the both versions of the real AMTI filter. The area with meteo-clutter we can find from the Doppler frequency plot as the wide area of nonzero Doppler shifts. In the power plots the blue and red curves represent the signal before and after filtration respectively. From here we can see that the both filters can suppress the both clutter types below the noise level. The input signal includes also a reflection of a moving aircraft. Its signal to noise ratio is principally not affected by the filtration, being about $20 \mathrm{~dB}$ at the filters output.

\section{CONCLUSION}

In this paper the design of the AMTI filter was described. Two new algorithms for coefficients computation for a real AMTI filter were introduced. The main advantage of the design with the "close roots" is a direct design of a filter with wide filter stopbands. The design with the "distant roots" needs a numerical optimization (Section 1.1.1) and leads to narrower stopbands. Finally, the cascaded AMTI filter was discussed. It was shown that it has not as a good characteristics as the previous one. In the last chapter the both designs of the real AMTI filter were tested with real measured data. It was verified that the both suggested filter designs work satisfactorily.

\section{Acknowledgment}

This study was supported by the grant of IGA University of Pardubice SGSFEI 2016.

\section{REFERENCES}

[1] BARTON, D. K.: Modern Radar System Analysis, Artech House, Norwood, MA, 1988.

[2] DAVÍDEK, V.: Analog and Digital Filters, 2nd edition, Vydavatelstv ČVUT, 2004.

[3] BARTSCH, H. J.: Math. Formulas (Matematické vzorce), 4th ed., Academia, Praha, 2006. (in Czech) 

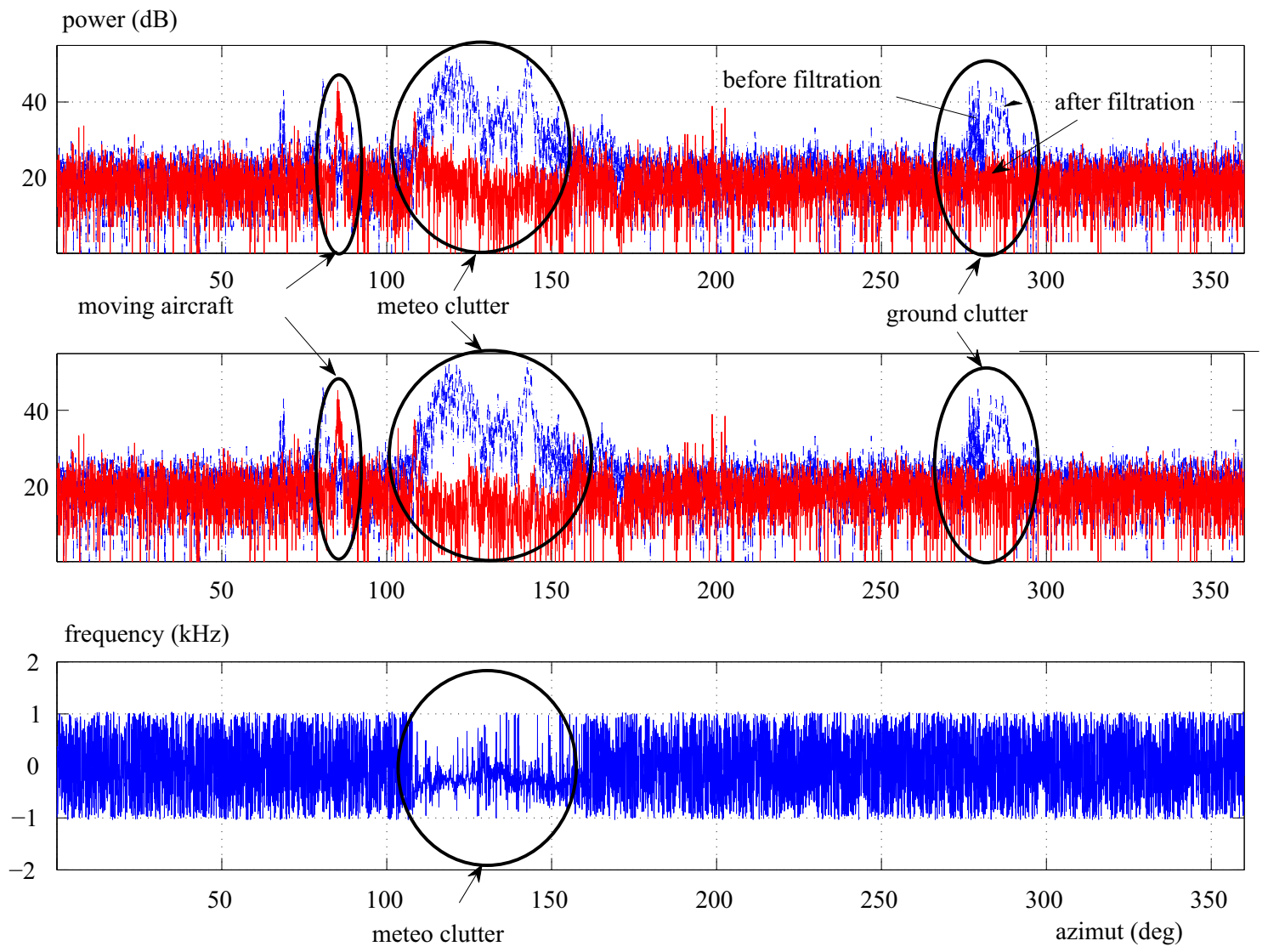

Fig. 14. Frequency response of a cascaded AMTI filter for staggered and non-staggered signals, from top down: AMTI with distant and closed roots, and the Doppler frequency

[4] MAHAFZA, B. R.-ATEF, Z. E. : MATLAB Simulations for Radar Systems Design, CRC Press/Chapman \& Hall, Boca Raton, FL, 2004.

[5] FRED, E. N.: Radar Design Principles: Signal Processing and the Environment, 2. ed., Scitech Publ, Raleigh, NC, 2004.

[6] SCHLEHER, D. MTI and Pulsed Doppler Radar: Artech House, Boston.

[7] MAHAFZA, B. R. : Radar Systems Analysis and Design using Matlab, Chapman \& Hall/CRC, Boca Raton, 2000.

Received 2015

Michal Řezníček was born in Pardubice, Czech Republic in 1991. He received his MS degree from the Faculty of Electrical Engineering and Informatics, University of Pardubice in 2015. Now he is pursuing his $\mathrm{PhD}$ with his thesis focused on signal processing in passive coherent radars.
Pavel Bezoušek was born in Ostrava, Czechoslovakia in 1943. He received his MS degree from the Czech Technical University in Prague in 1966 and his $\mathrm{PhD}$ degree from the same university in 1980. He was with the Radio Research Institute of the Tesla Pardubice from 1966 till 1994, where he was engaged in the microwave circuits and systems design. Since then he is with the University of Pardubice, now at the Faculty of Electrical Engineering and Informatics. Presently he is engaged in the design of radar systems.

Tomáš Zálabský was born in Pardubice, Czech Republic in 1988. He received his MS degree from the Faculty of Electrical Engineering and Informatics, University of Pardubice in 2012. Now he goes on in postgradual study focused on signal processing in frequency modulated continuous wave radars and design of antennas for a radars. 